\title{
Case report: management of cystic adenomyosis
}

\begin{abstract}
Adenomyosis has a negative impact on fertility owing to reduced likelihood of clinical pregnancy and implantation and increased risk of early pregnancy loss. Ultrasound detection of adenomyotic changes include globular uterine enlargement, wall thickening, linear striations, thickened endomyometrial borders, junctional zone and cystic anechoic spaces in myometrium. The aim of the case report is to present hysteroscopic dissection and ablation of subendometrial adenomyotic cyst with good subsequent ART outcome.
\end{abstract}

Volume 5 Issue 4 - 2019

\section{Shilpa Saple, Mukesh Agrawal, Simi Kawar}

Aarush IVF and Endoscopy Centre, India

Correspondence: Shilpa Saple, Aarush IVF and Endoscopy Centre, India, Tel 9819930643, Email drshilpasaple@gmail.com

Received: May 18, 2019 | Published: July 05, 2019

\section{Introduction}

Our objective is to present hysteroscopic dissection and ablation of adenomyotic cysts as a method of surgical management of this condition and discuss the implications and treatment in an infertile patient. Adenomyosis by definition is the benign invasion of endometrium into the myometrium producing a diffusely enlarged uterus which microscopically exhibits ectopic, non-neoplastic endometrial glands and stroma surrounded by hypertrophic and hyperplastic myometrium. Although it has been a histopathological diagnosis, with current modalities of 2D, 3D sonography and MRI, it is possible to diagnose this condition in vivo.

\section{Diagnosis on TVS scan by the following distinctive features}

Asymmetrical myometrial thickening, parallel shadowing, myometrial cysts, hyperechoic islands, irregular endo-myometrial junction. On 3D USG, junctional zone-thickening and disruption in adenomyosis, under normal circumstances it is hypoechoeic, heterogenous myometrial echotexture, increased echogenicity or linear striation due to ectopic endometrial tissue and presence of Subendometrial cysts. MRI criteria -thickness of Junctional zone $>12 \mathrm{~mm}$, broadening of JZ is infiltrative (normal $<5 \mathrm{~mm}$ ). High T2 signal intensity linear striations radiating out of the endometrium. ${ }^{1,2}$

\section{Distinctive features of differentiation from fibroid are as in chart below}

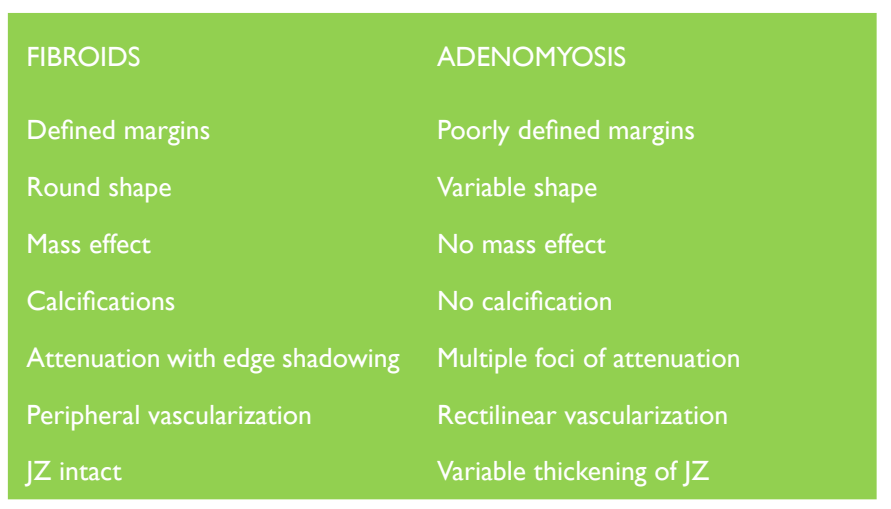

\section{Brosens classification of cystic adenomyosis (MUSCLE)}

a. M, myometrial location (intramural, submucous, subserous)

b. U, uterine site (midline,paramedian, lateral)

c. S, structure (cystic, mixed, polypoid)

d. C, contents (clear, hemorrhagic)

e. L, level (fundus, body, cervix)

\section{Effects of adenomyosis on infertility}

I. Altered peristaltic activity- responsive to endocrine and paracrine stimuli - Estrogen due to aromatase P450 overexpression, conversion of androgen to estrogen and oxytocin, PGs, GF, cytokines $\rightarrow$ dysperistalsis by Kunz.

II. Altered endometrial function and receptivity-Colonisation of endometrium with macrophages by Leiva.

III. Impaired implantation -expression of integrins and pinopode formation is affected.

IV. Altered decidualisation overexpression of cytochrome P450 $\rightarrow$ estrogen receptor\& lack of PR.

V. Abnormal concentration of intrauterine free radicals -Nitic oxide, superoxide.

VI. Gene dysregulation.

VII. High risk of miscarriage.

\section{Case report}

Mrs. X 32-year-old was referred to us as case of primary infertility of 5 years duration. She had regular and painful menses. Husband's Semen analysis was normal. Her HysteroLaparoscopy 3 years back was done which had normal findings and she had undergone 2 cycles of IUI with Clomifene stimulation previously without any resulting pregnancy.

\section{General examination was normal}

Positive findings on TVS- Transverse dimensions were increased. Presence of 2 intramural subendometrial cysts of $1 \mathrm{~cm}$ and $0.8 \mathrm{~cm}$ 
above the cervix on the posterior wall adjacent to each other and indenting the cavity. Her antral follicular count was 11 . Her routine investigations and infection screen were done which were normal and AMH was 2.6. She was given one cycle of HMG 150 units $\mathrm{x} 10$ days from $2^{\text {nd }}$ day of menses and trigger at follicular size of $19-20 \mathrm{~mm}$ and IUI was done on $12^{\text {th }}$ day with progesterone support post IUI. The cycle was negative. She was counselled for an ICSI cycle and precycle hysteroscopy was planned. Under General anesthesia, a diagnostic hysteroscopy was done using $2.9 \mathrm{~mm}$ hysteroscope with Bettocchi operating sheath connected to a HD 3 chip Storz camera with saline distention using hysteromat. Uterine cavity was normal and a bulge of $1 \mathrm{~cm}$ was seen on the posterior wall just above cervix. Using the same scope mounted on a bipolar resectoscope assembly using normal saline as a distending medium, a linear incision was made over the bulge with a Collin's knife. ${ }^{3-5}$

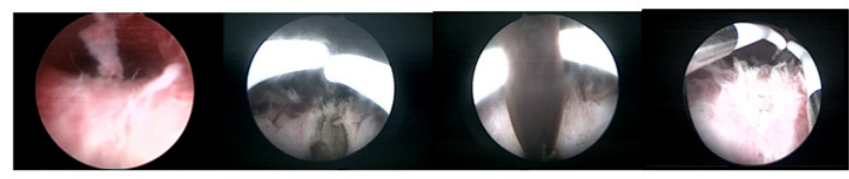
a. bulge on posterior wall
b. linear incision by Collins knife
c. chocolate fluid
d. base of the cyst

ICSI was planned with long protocol.

A. Lupride $0.5 \mathrm{mg}$ starting 7 days post ovulation in the same cycle.

B. Stimulation with 300 units HMG was carried out after adequate downregulation (I0days).

C. $6 \mathrm{M} 2$ eggs were obtained and transfer of 3 embryos on Day 3 yielded a positive Bhcg test.

\section{Differential diagnosis}

a) Cystic degeneration in fibroid

b) Congenital anomaly- hematometra of horn

\section{Other modalities of treatment}

i. Scissors - open and resect the lesion with base.

ii. Fulgurate base with ball electrode

iii. Laparoscopic approach can be tried if lesions are in outer intramural part

iv. USG guided aspiration for large lesions

v. Spirotome

vi. Robotics

\section{Conclusion}

Hysteroscopic evaluation of the endometrial surface can detect changes, subtle lesions of which the pathological value is not yet proven but can be described as possible although not pathognomonic signs of adenomyotic changes in the myometrium. Endometrial changes like hyper-vascularization, strawberry pattern, endometrial defects and submucosal hemorrhagic cysts are suggestive of adenomyosis $(78,80,81)$ (Figure 1). A cystic translucent area in the fundal area visualized by TVS, appearing as a bulging structure in the uterine cavity was described. Biopsy of the bed of the cyst was on histology diagnosed as adenomyosis (81). With the increasing evidence of the importance of the inner myometrium, uterine exploration in patients with infertility, abnormal uterine bleeding and pain should not be restricted to exploration of the uterine cavity but should include the exploration of the inner and outer myometrial structures.

1. Adenomyotic sub endometrial cyst $>5 \mathrm{~mm}$, which can be picked up as a bulge on hysteroscopy can be drained and base cauterized to improve the pregnancy chances.

2. Hysteroscopy - clear visualization of intracavitary lesions with direct access

3. Treatment by mechanical dissection or bipolar ablative surgery produces minimal tissue damage.

4. Further studies would be required to understand its impact on fertility and benefits of its surgical removal.

\section{Acknowledgments}

None.

\section{Conflicts of interest}

The author declares there are no conflicts of interest.

\section{References}

1. Gordts S, Campo R, Brosens I. Hysteroscopic diagnosis and excision of myometrial cystic adenomyosis. Gynecol Surg. 2014;11:273-278.

2. Hiroyuki Takeuchi, Mari Kitade, Iwaho Kikuchi, et al. Diagnosis, laparoscopic management, and histopathologic findings of juvenile cystic adenomyoma: a review of nine cases. Fertility and Sterilityâ. 2010;94(3):863-868.

3. Mi Ju Kim. A case of cystic adenomyoma of the uterus after complete abortion without transcervical curettage. Obstet Gynecol Sci. 2014;57(2):176-179.

4. Paolo Vercellini, Dario Consonni, Dhouha Dridi, et al. Uterine adenomyosis and in vitro fertilization outcome: a systematic review and meta-analysis. Hum Reprod. 2014;29(5):964-977.

5. Sebastiano Campo, Vincenzo Campo, Giuseppe Benagiano. Adenomyosis and infertility. Reproductive Biomedicine. 2012;24:35-46. 